\title{
Estimation of Double Smoothing Parameters with Simulation
}

Fadel A. Al-Taie

Statistics Department/College of Computer Science and

Mathematics/University of Mosul, Iraq

\section{Received on: 01/03/2004}

Accepted on: 22/03/2004

\section{Abstract}

In this paper we estimate that the equation of smoothing to the first Smoothing is the same equation of double exponentioal smoothing, and finding the equation of prediction of two state first: the variance of the original time cerise $\mathrm{x}_{\mathrm{t}},\left(\sigma_{\mathrm{xt}}^{2}\right)$ is the same as the variance of the $\mathrm{Ft},,\left(\sigma_{\mathrm{Ft}}^{2}\right),\left(\sigma_{\mathrm{xt}}^{2}=, \sigma_{\mathrm{Ft}}^{2}\right)$, and the second state the variance of $x_{t},,\left(\sigma_{x t}^{2}\right)$ is not the same as the variance of $\mathrm{Ft}, \sigma_{\mathrm{ft}}^{2},\left(\sigma_{\mathrm{xt}}^{2}=, \sigma_{\mathrm{Ft}}^{2}\right) . \mathrm{Ft}$

This results will be applied to the real data which represent the mean of relative wet of Mosul city, and also to the data obtained form simulation, with use the $\left(\chi^{2}, \mathrm{MSD}\right)$ to select the best of smoothing.

Keywords: Parameter of smoothing, exponential smoothing, prediction, variance, simulation, relative wet.

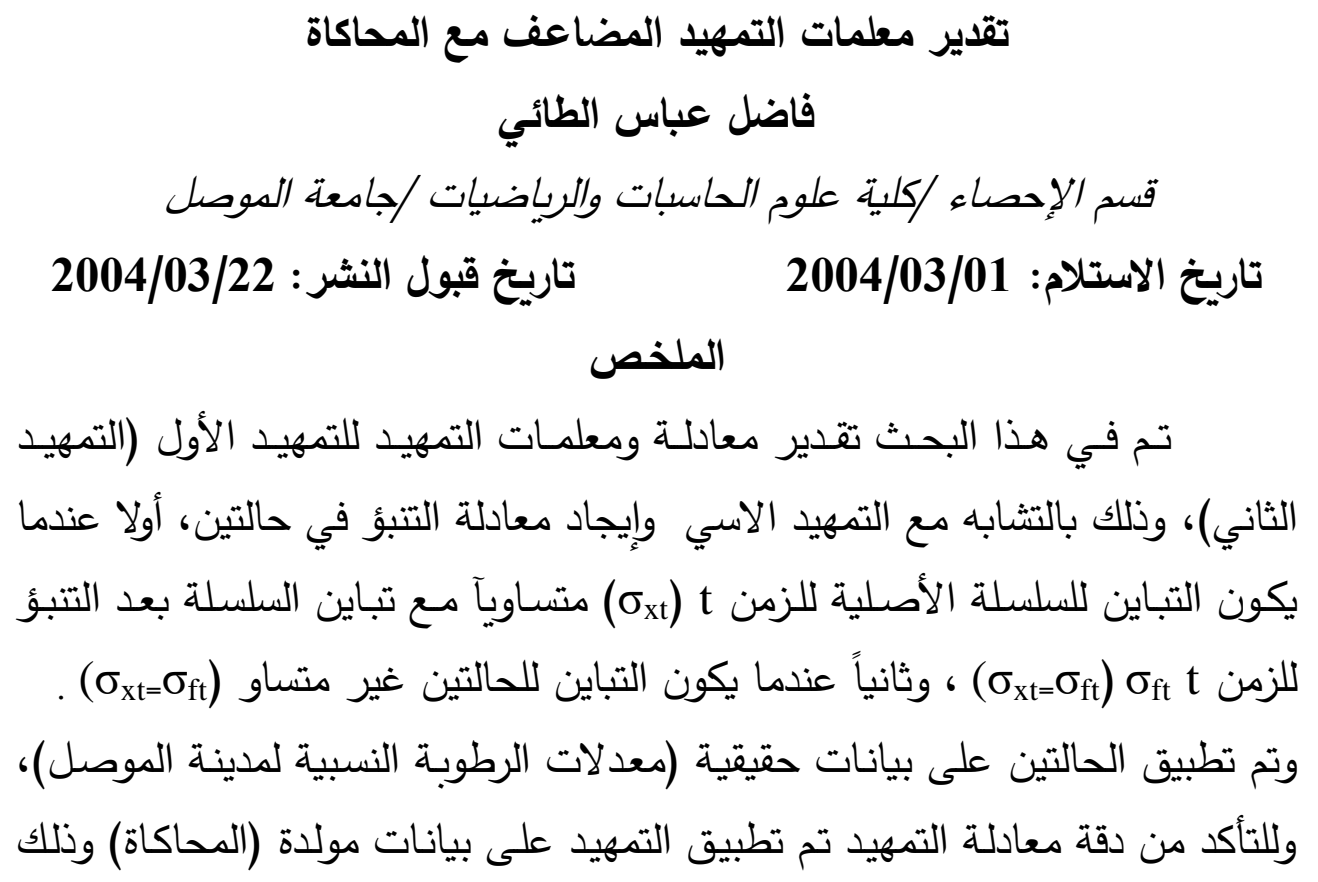


بتوليد بينات بأعداد (80، 120، 200) وتم استخدام المقاييس الإحصائية مربع كاي ومتوسط مربعات الخطاء ( MSD ; X2 ) لغرض التأكد من دقة التمهيد والمفاضلة

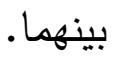

الكلمات المفتاحية: معلمات التمهيد، التمهيد الاسي، تباين، التتبؤ، المحاكاة، الرطوبة

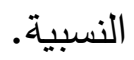

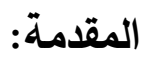

إن الاعتماد على البيانات الموجودة والمعومات السابقة من الإجراءات المهمة جداً في عمليـة التقدير التي أصبحت من أهم الأمور في علم الإحصـاء وأكثرهـا

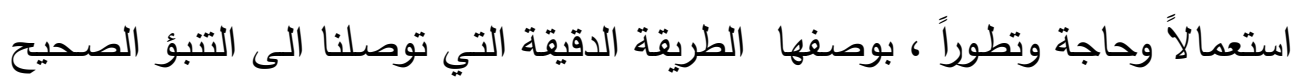

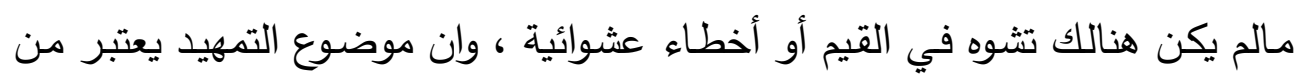

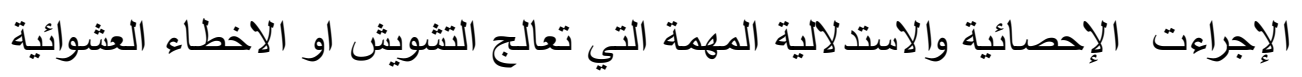

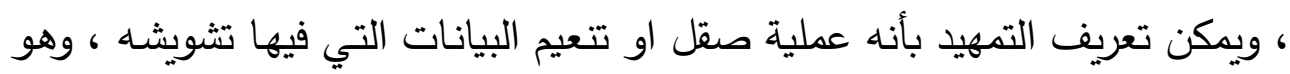

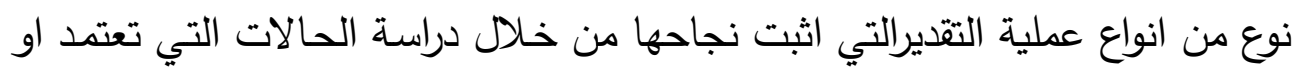

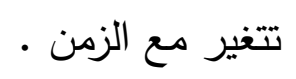

وفي بحثا هذ تمت دراسة التمهيد الثاني (التمهيد للتمهيد الاول) او ممكن

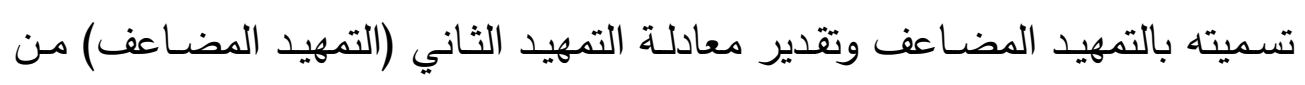
خلال تقدير المعلمات او اعادة التقدير من خـلال توافر البيانات من التمهيد الاول

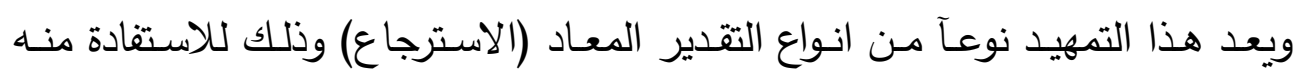

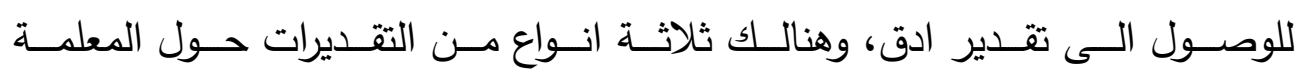

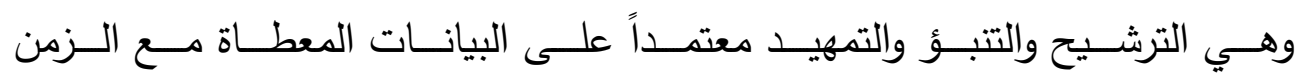
( x $\left.1, x_{2}, \ldots, x_{t}\right) t$

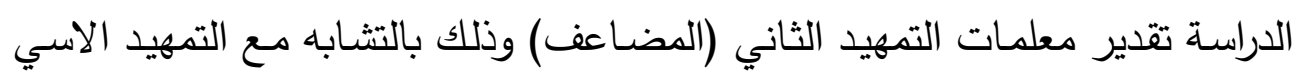

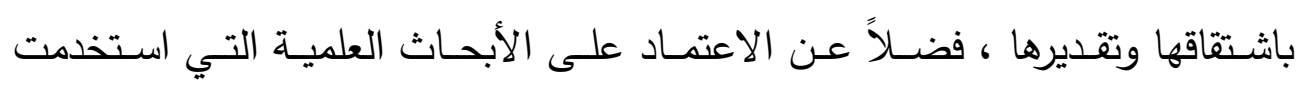

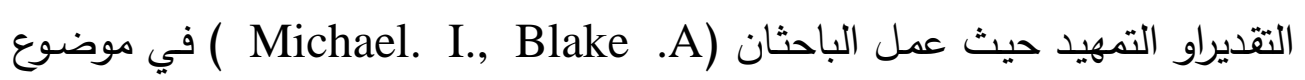


التمهيد (Smoothing filter for Condensation) وكنلــك عمـل الباحثون( Celia.F,Balaji.V,Les.M, \&Amar.R) وتضمن بحثهم ربط التمهيد

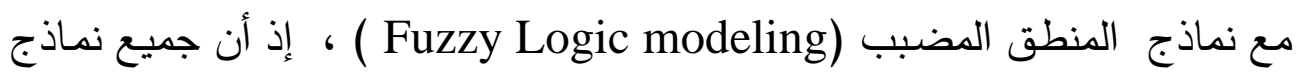

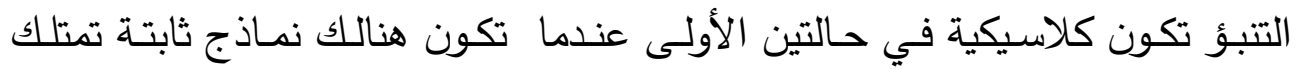
معلمات ثابتة وتباينا ثابتا، وان هذه الحالة تتضمن او تثمل شرح كل طرائق التتبؤ

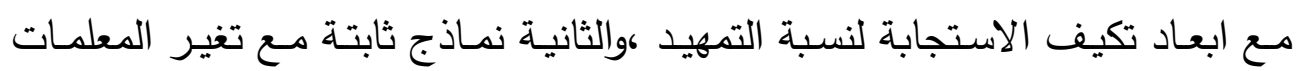

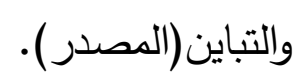

اذ حيـث ان التقدير الإحصـائي الجيد دائهـاً يحساول تصـغير قيمـة التبـاين

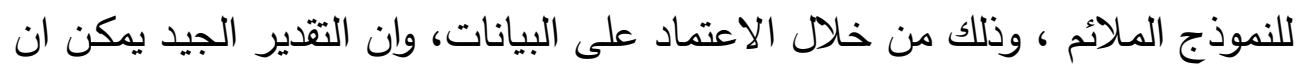

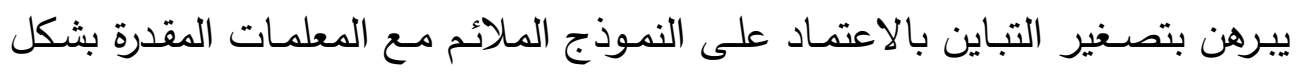

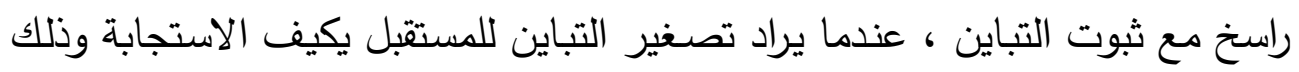

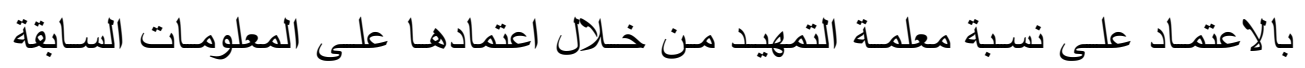

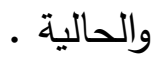
الإهف :

يهذف البحث الى إيجاد معلمات معادلة التمهيد الثاني وتقديرها ذلك بالتشابه مـع التمهيد الآسي و تطبيق ذلك على بيانات حقيقيـة معدلات الرطوبـة النسبية

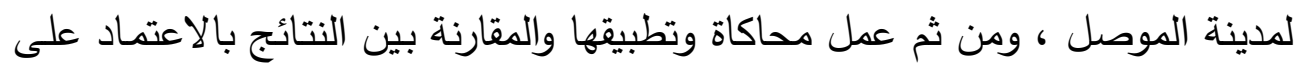

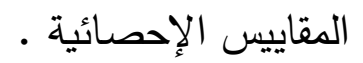




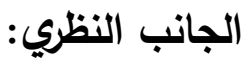

تمهيد البيانات البسيطة Smoothing univariate Data

إذا كان Xt تمثل السلسلة الزمنية للبيانات الحقيقية او المعلومات المتوافرة و

هي التتبؤ للسلسلة الزمنية ، فان التتبؤ للتمهيد الاول في الفترة t+1 يمكن التعبير عنه وذلك بالتشابه مع التمهيد الاسي بالمعادلة آلاتية : $F_{t+1}=w x_{t}+(1-w) F_{t}$

وان تباين $\sigma_{F t+1}^{2}=(1-w)^{2} \sigma_{F}^{2}+w^{2} \sigma_{x}^{2}$

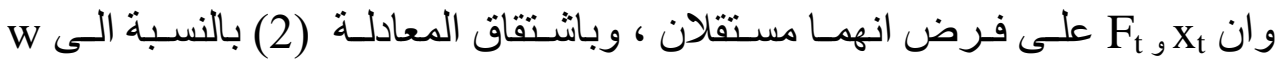
نحصل على المعلمة W ) هو ثابت التمهيد Smoothing constant) مـ خـلال

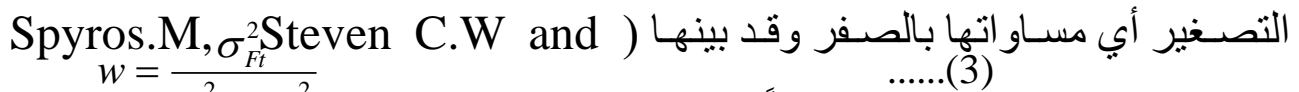
$w=\frac{\sigma_{F t}^{2}}{\sigma_{F t}^{2}+\sigma_{x}^{2}}$

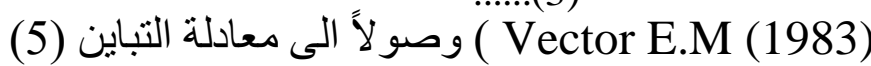

من ثم نعوض بقيمة W في المعادلة (1)

$F_{t+1}=\frac{\sigma_{F t}^{2}}{\sigma_{F t}^{2}+\sigma_{x}^{2}} x_{t}+\left(1-\frac{\sigma_{F}^{2}}{\sigma_{F t}^{2}+\sigma_{t x}^{2}}\right) F_{t}$

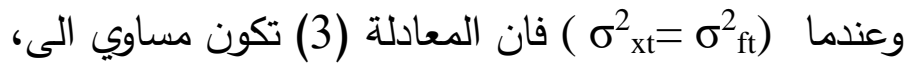

$w=\frac{\sigma_{F t}^{2}}{\sigma_{F t}^{2}+\sigma_{x t}^{2}}=\frac{1}{2}$

وان التباين الكلي نحصل عليه بعد تعويض قيمة (w) في المعادلة (2) كما هو

$\sigma_{F+1}^{2}=\left(1-\frac{\sigma_{F t}^{2}}{\sigma_{F t}^{2}+\sigma_{x}^{2}}\right)^{2} \sigma_{F}^{2}+\left(\frac{\sigma_{F}^{2}}{\sigma_{F}^{2}+\sigma_{x}^{2}}\right)^{2} \sigma_{x t}^{2}$

الأتي 
وان فكرة هذا البحث هو اخذ التمهيد للمعادلة (1)

$$
F_{t+1}=w x_{t}+(1-w) F_{t}
$$

والتي تعتمد على التثـابه مـع التمهيد الاسي ، عندما نأخذ التمهيد للمعادلة (1) تكون بالثكل الأتي تعني

$$
F_{t+1}^{\prime}=w F_{t+1}+(1-w) F^{\prime}
$$

التمهيد للتمكيد الاول في الفترة $F_{t+1}^{\prime}=(t+1)$

التمهيد الاول في الفترة(t+1

التنبؤ في الفترة(t)

W= ثابت التمهيد

وبتبسيط المعـادلة (6) وذلك بتعويض قيمة التمهيد الاول معادلة (1) في المعادلة

$F_{t+1}^{\prime}=w\left[w x_{t}+(1-w) F_{t}\right]+(1-w) F_{t}^{\prime} \Rightarrow F_{t+1}^{\prime}=w^{2} x_{t}+w(1-w) F_{t}+(1-w) F_{t}^{\prime}=\mathrm{Ft}+1 \ldots(7)$

ولغرض الحصول على نسبة التمهيد (w) من هذه المعادلة لا يمكننا استخدام طريقة

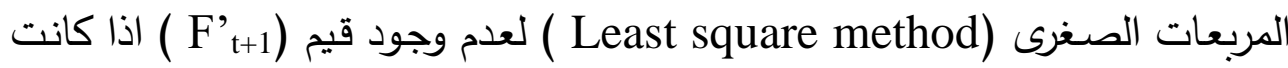
المعادلة خطية وكذلك لايمكن استخدام طريقة Marquartede اذا كانت غير خطية

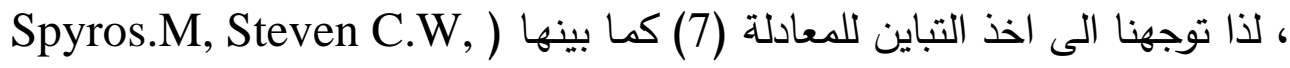

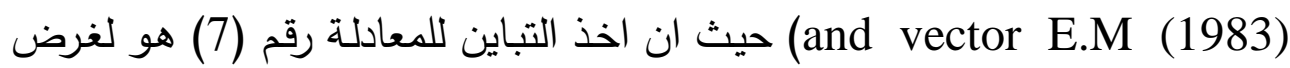
الحصول على نسبة التمهيد التي تعتمد على تباين البيانات الأصلية وكذلك تباين

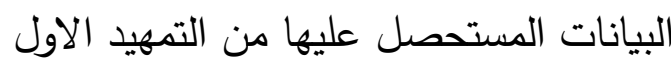


وعندما نأخذ التباين للمعادلة (7) نحصل على (8)، استتاداً إلى فرضيتنا السابقة انهما مستقلان

$\sigma_{t+1}^{2}=w^{4} \sigma_{x}^{2}+w^{2}(1-w)^{2} \sigma_{F}^{2}+(1-w)^{2} \sigma_{F}^{2}$

$$
\sigma_{t+1}^{2}=w^{4} \sigma_{x}^{2}+w^{2} \sigma_{F}^{2}-2 w^{3} \sigma_{F}^{2}+w^{4} \sigma_{F}^{2}+\sigma_{F}^{2}-2 w \sigma_{F}^{2}+w^{2} \sigma_{F}^{2} .
$$

$$
\frac{\partial \sigma^{2}}{\partial w}=4 w^{3} \sigma_{x}^{2}+2 w \sigma_{F}^{2}-6 w^{2} \sigma_{F}^{2}+4 w^{3} \sigma_{F}^{2}-2 \sigma_{F}^{2}+2 w \sigma_{F}^{2}
$$

وبتبسيط المعادلة (8) نحصل على

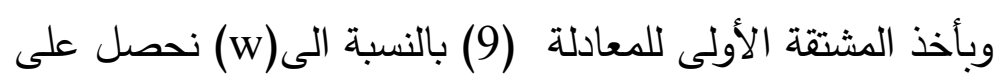

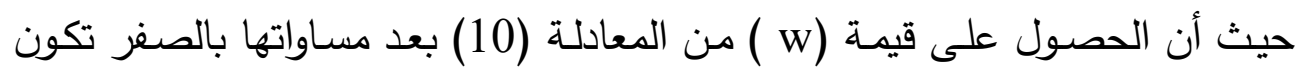

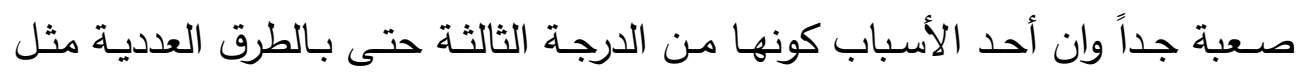

$$
\frac{\partial^{2} \sigma^{2}}{\partial w^{2}}=12 w^{2} \sigma_{x}^{2}+2 \sigma_{F}^{2}-12 w \sigma_{F}^{2}+12 w^{2} \sigma_{F}^{2}+2 \sigma_{F}^{2}
$$

طريق لاكرانج والقيم التي نحصل عليها قد تكون خيالية، لذا تم التوجه الى اخذ المشتقة للمعادلة (10 ) حيث -نحصل ولى على المعادلة (11)

$$
\begin{aligned}
& 12 w^{2} \sigma_{x}^{2}+2 \sigma_{F}^{2}-12 w \sigma_{F}^{2}+12 w^{2} \sigma_{F}^{2}+2 \sigma_{F}^{2}=0 \\
& \text { بمساواة المعاداة (11) بالصفر لغرض الحصول على نسبة التمهيد } \\
& w^{2}\left(\sigma_{x}^{2}+\sigma_{F}^{2}\right)-w \sigma_{F}^{2}+\frac{1}{3} \sigma_{F}^{2}=0
\end{aligned}
$$

تم حل هذه المعادلة بطريقة الدستور وتم الحصول على نسبة التمهيد(W) 
$w=\frac{\sigma_{F}^{2} \mp \sqrt{\sigma_{F}^{4}-2\left(\sigma_{x}^{2}+\sigma_{F}^{2}\right) \sigma_{F}^{2}}}{2\left(\sigma_{x}^{2}+\sigma_{F}^{2}\right)}$

ان نتيجة هذه المعادلة ممكن تعويضها في المعادلة (7) كنسبة التمهيد ولكن قد تكون هذه النسبة أحيانـا تعطي نتيجـة خيالية ، لذا تـم التوجـه الى اخذ مشتقة مـرة أخرى للمعادلة

$\frac{\partial^{3} \sigma^{2}}{\partial w^{3}}=24 w \sigma_{x}^{2}-12 \sigma_{F}^{2}+24 w \sigma_{F}^{2}$

$24 w \sigma_{x}^{2}-12 \sigma_{F}^{2}+24 w \sigma_{F}^{2}=0$

$$
w=\frac{12 \sigma_{F}^{2}}{24 \sigma_{x}^{2}+24 \sigma_{F}^{2}}=\frac{\sigma_{F}^{2}}{2\left(\sigma_{x}^{2}+\sigma_{F}^{2}\right)}
$$

وبمساواة المعادلة بالصفر نحصل على

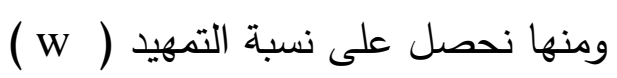

وبتعويض المعادلـة (13) في المعادلـة (6) نحصـل على معادلـة التمهيد للتمهيد الاول • وتم التحقق من صحة التمهيد في الفقرة (التمهيد الاول والتمهيد للتمهيد الاول) بحالتين:

$$
\left(\sigma_{\mathrm{xt}}^{2}=\sigma_{\mathrm{Ft}}^{2}\right) \text { عندمان } 1
$$

$F_{t+1}^{\prime}=\frac{\sigma_{F}^{2}}{2\left(\sigma_{x}^{2}+\sigma_{F}^{2}\right)} F_{t+1}+\left(1-\frac{\sigma_{F}^{2}}{2\left(\sigma_{x}^{2}+\sigma_{F}^{2}\right)}\right) F_{t}^{\prime}$

$F_{t+1}^{\prime}=\frac{1}{4} F_{t+1}+\frac{3}{4} F_{t}^{\prime}$

تكون المعادلة بالثكل الأتي ( $\left.\sigma_{\text {xt }}^{2}=, \sigma_{\mathrm{ft}}^{2}\right)-2$ وان التباين للفترة t+1 في الحالتين $\left(\sigma_{\mathrm{xt}}^{2}=\sigma_{\mathrm{ft}}^{2}\right)$ عندما 1

$\sigma_{F t+1}^{2}=\left(1-\frac{\sigma_{F t}^{2}}{2\left(\sigma_{x t}^{2}+\sigma_{F}^{2}\right)}\right)^{2} \sigma_{F}^{2}+\left(\frac{\sigma_{F}^{2}}{2\left(\sigma_{x}^{2}\left(\boldsymbol{G \zeta}_{F}^{2}\right)\right.}\right)^{2} \sigma_{F t+1}^{2}$ 


$$
\left(\sigma_{\mathrm{xt}}^{2}=\sigma_{\mathrm{ft}}^{2}\right) \text { عندما } \quad-2
$$

\section{مقارنة بين التمهيد الاول والتمهيد للتمهيد الاول}

$$
\sigma_{F t+1}^{2}=\left(\frac{3}{4}\right)^{2} \sigma_{F t}^{2}+\left(\frac{1}{4}\right)^{2} \sigma_{F t+1}^{2}
$$

ولغـرض مقارنـة التمهيد الاول مـع التمهيد للتمهيد الاول تم الاعتمـاد على التباين للحالتين وذلك على وفق معادلتي التباين

$$
\begin{aligned}
& \sigma_{F+1}^{2}=\left(1-\frac{\sigma_{F}^{2}}{\sigma_{F t}^{2}+\sigma_{x}^{2}}\right)^{2} \sigma_{F}^{2}+\left(\frac{\sigma_{F}^{2}}{\sigma_{F}^{2}+\sigma_{x}^{2}}\right)^{2} \sigma_{x t}^{2} \\
& \sigma_{F t+1}^{2}=\left(1-\frac{\sigma_{F}^{2}}{2\left(\sigma_{x}^{2}+\sigma_{F}^{2}\right)}\right)^{2} \sigma_{F}^{2}+\left(\frac{\sigma_{F}^{2}}{2\left(\sigma_{x}^{2}+\sigma_{F}^{2}\right)}\right)^{2} \sigma_{F t+1}^{2}
\end{aligned}
$$

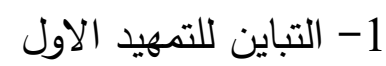

2- التباين للتمهيد للتمهيد الاول الاول

1 - عندما

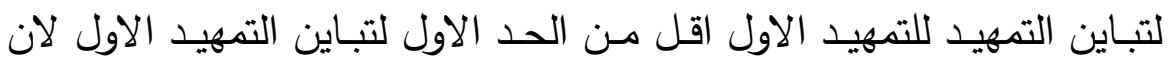
تباين التمهيد للتمهيد الاول يمثل التنبؤ لقيم السلسلة بعد اجراء التمهيد الاول

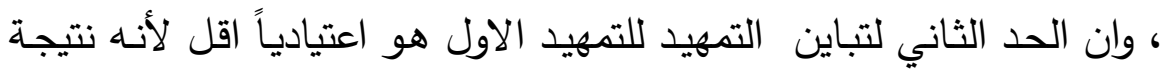

$$
\sigma_{F+1}^{2} \leq \sigma_{F+1}^{2}
$$

التمهيد الاول أي تم صقل البيانات بها وبتعبير أخر تم تتعيم القيم الثـادة

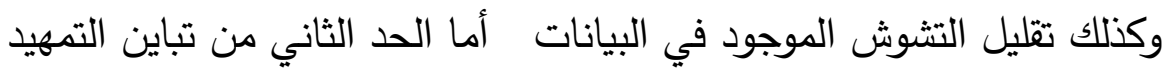

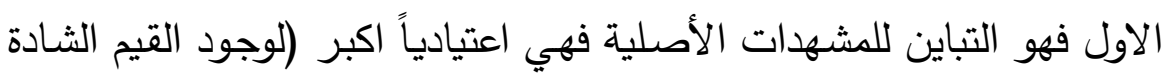
والأخطاء العشوائية) من التباين قبل التمهيد وبهذه الحالة يكون تباين التمهيد للتمهيد الاول اقل من تباين التمهيد الاول وهذا مما يدل على صحة التمهيد

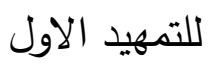


2 - عندما

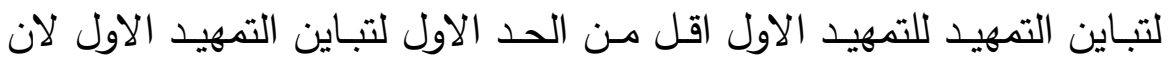

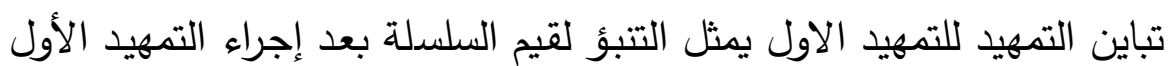

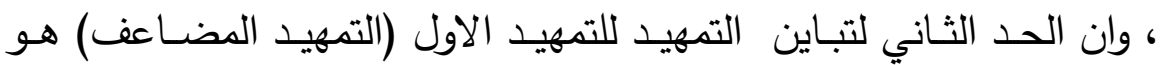

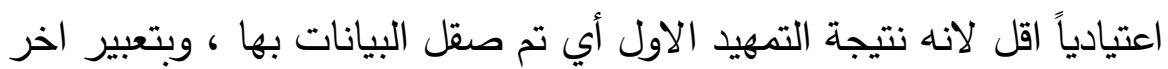

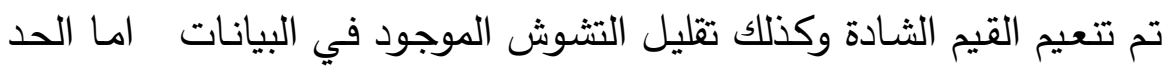
الثاني من تباين التمهيد الاول فهو التباين للمشهدات الاصلية فهي اعتيادياً اكبر (لوجود القيم الثادة والأخطاء العشوائية) من التباين قبل التمهيد وبهن لتهنه

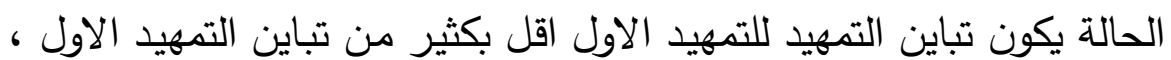

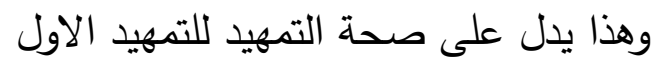
$\sigma_{F+1}^{2} \leq \sigma_{F+1}^{2}$

\section{قياس صحة ملاءمة التمهيد}

1- لاختبار ملائمة التمهيد للسلسلة الزمنية لحالتي التمهيد الاول والتمهيد للتمهيد

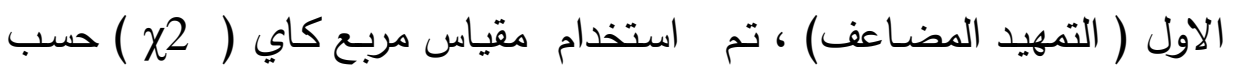
$\chi^{2}=\sum_{i=1}^{n} \frac{\left(O_{i}-E_{i}\right)^{2}}{E_{i}}$

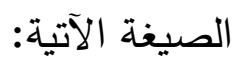

$$
\text { حيث أن }
$$

Ei تمثل القيم الحقيقية او المشاهدة Oi

وان n عدد المشاهدات ، حيث تقارن قيمة (22 ) الدحسوبة مع الجدولية بدرجة حرية

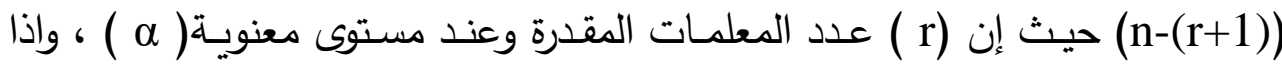

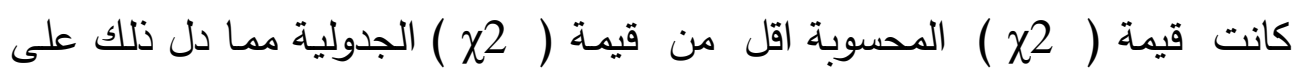

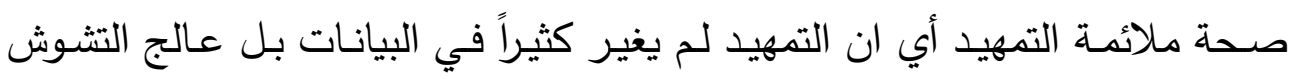


الموجود في البيانات ، وتم التاكد من صحة التمهيد الاول والتمهيد للتمهيد الاول للبيانات المولدة بالمحاكاة ايضاً.

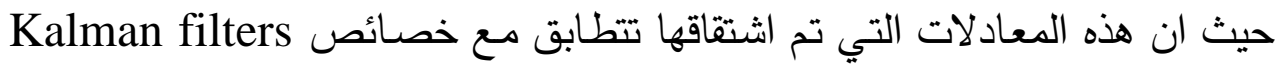
على وفق النقاط الآتية : (Spyros.M, \& els) 1- التقديرات المتطورة هي التي قدرت بواسطة الاعتمـاد على البيانات القديمة

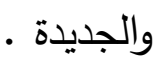
2- ان الاوزان قدرت من خـلال الاعتماد على البيانات القديمة والجديدة وكذلك

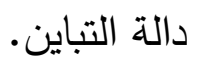
3- ان المعلمـات المقدرة والتبـاين يمكن ان تحسب بالاستعانة بخصـائص Kalman filters 4- - هنالك بعض الصعوبة في حساب التباين للتمهيد الثاني.

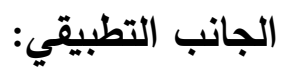

تتضمن دراستتا التطبيقية معدلات الرطوبـة النسبية لمحافظة نينوى للفترة (1941-

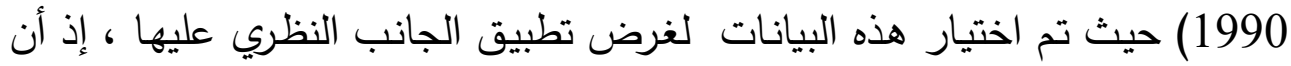
إجراء التمهيد الأول للبيانات هو الخطوة الأولى من عملية المعالجة (تتعيم البيانات)

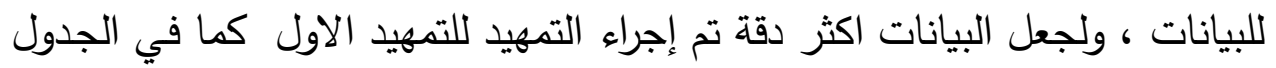

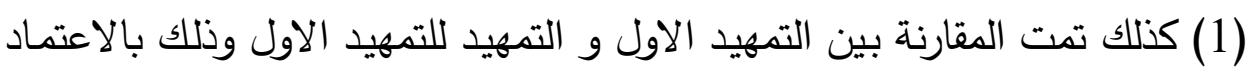

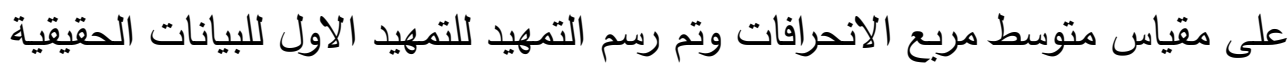

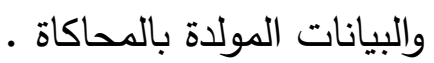

Mean Squared Deviation ( MSD متوسط مربع الانحرافات

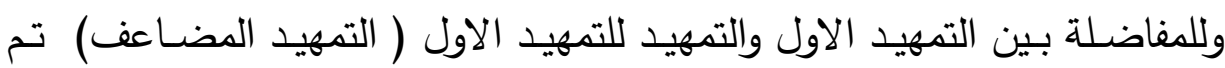
استخدام متوسط مربع الانحرافات اذ كلما كانت قيمته اقل دل على دقة الته التمهيد 
$M S D=\frac{\sum_{t=1}^{n}\left(y_{t}-y_{t}\right)^{2}}{n}$

حيث ان (y ) تمثل القيم المشاهدة ، و y y ) تمثل القيم بعد إجراء التمهيد للبيانات ،

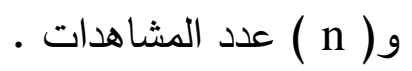


تقدير معاديلة التمـيد

1- لقد تم تقدير معادلة التمهيد الاول بالاعتماد على المعادلة رقم (3) وكانت

معادلة التمهيد الاول

$F_{t+1}=0.182 x_{t}+0.818 F_{t_{t}}$

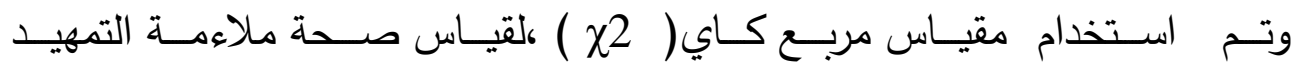

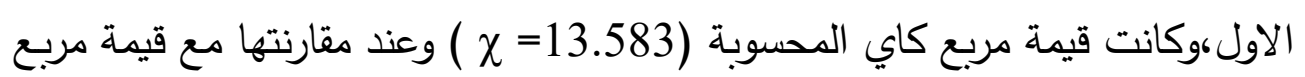

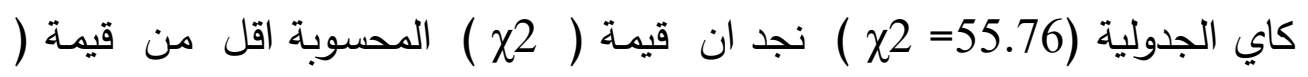

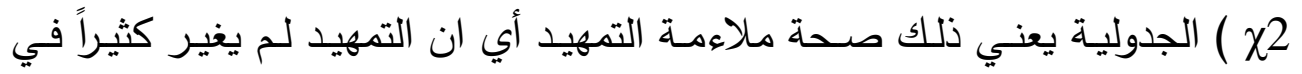
البيانات بل عالج التشويش الموجود في البيانات .

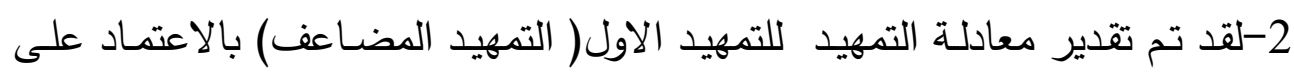
المعادلة رقم (13) وكانت معادلة التمهيد للتمهيد الاول

$F_{t+1}^{\prime}=0.1976 x_{t}+0.803 F_{t_{t}^{\prime}}^{\prime}$

وتم استخدام مقياس مربع كاي(2)، لقياس صحة ملاءمـة التمهيد للتمهيد الاول،

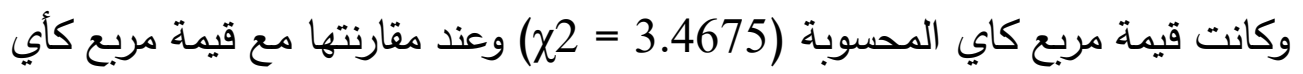

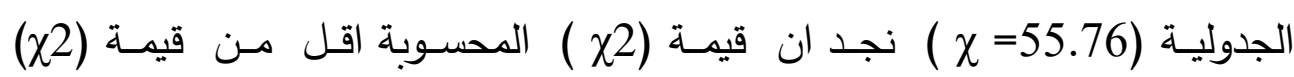

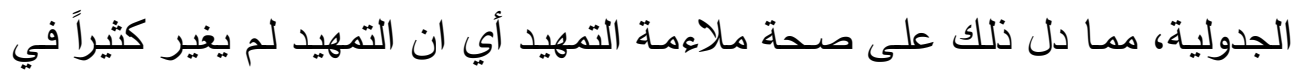

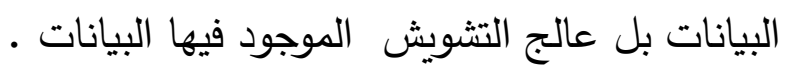

\section{متوسط مربع الانحرافات( MSD)}

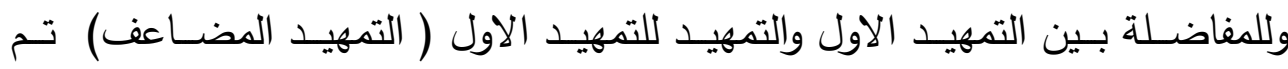

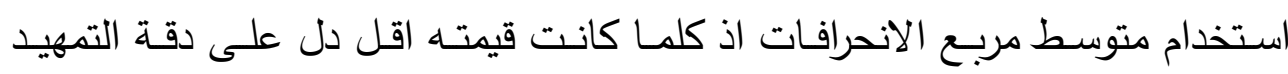

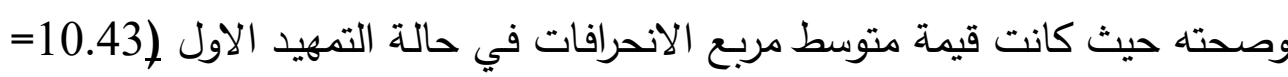

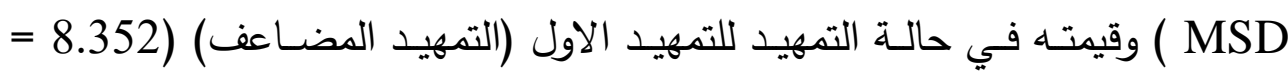
) وعند المقارنة بين القيمتين نجد ان التمهيد المضاعف افضل من التمهيد 
الاول، ، والثكل (1) ، يبين قيم التمهيد للتمهيد الاول(التمهيد المضـاعف) وقيم

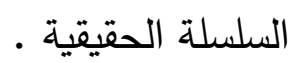

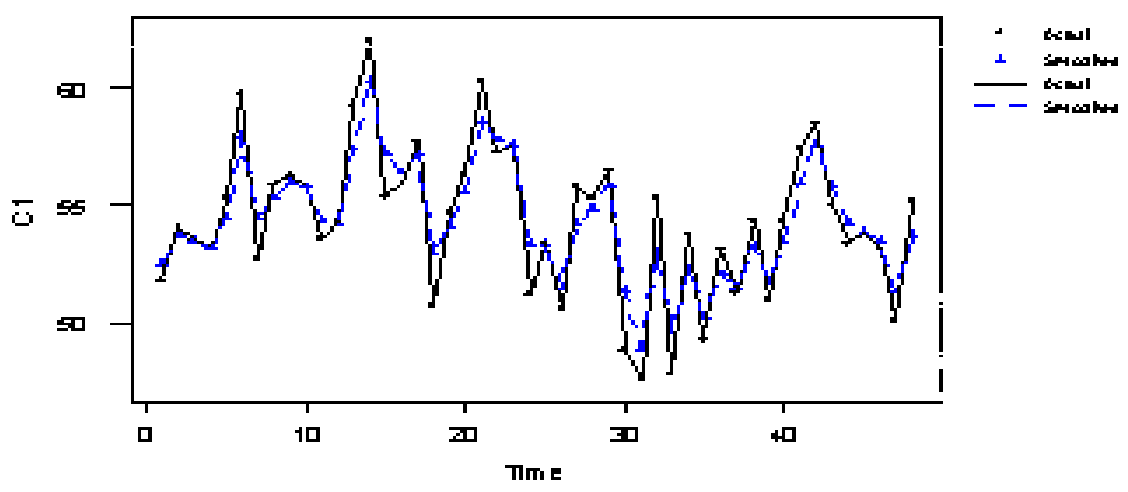

الثكل (1) يبين قيم التمهيد للتمهيد الاول (التمهيد المضاعف)

وقيم السلسلة الحقيقية .

المحاكاة : (المان

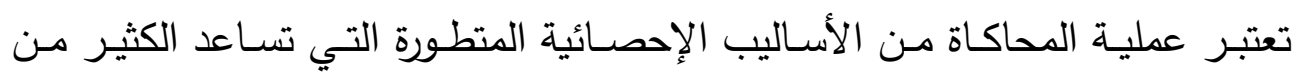

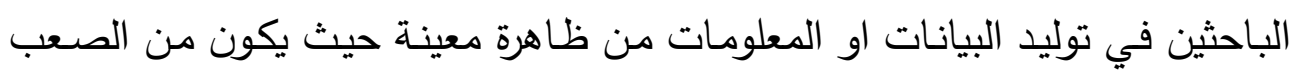
في الكثير من الظواهر الحصول على البيانات وفي حالة عدم الحصول على فلى البيانات

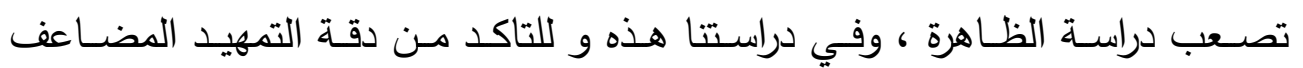

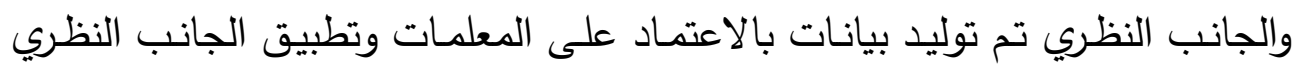

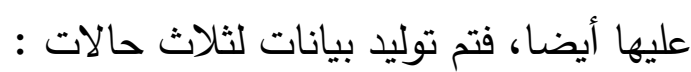

1- تم توليد بيانات بعدد 80 وذلك بنفس التباين والمعدل للبيانات الحقيقية ومن تم

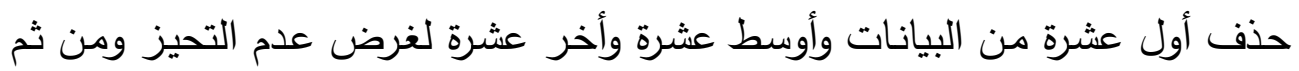
تطبيق الجانب النظري عليها وكان معادلة التمهيد لها 
$F_{t+1}^{\prime}=0.2976 F_{t+1}+0.703 F_{t}^{\prime}$

حيث كانت قيمـة مربع كاي المحسوبة (5.7109=2ح) وعند مقارنتها مـع الجدولية (2=55.76) نجدها ايضـاً اقل من الجدولية ممـا يدل على صحة التمهيد وكانت قيمـة متوسط مربعات الخطـاء (MSD=17.438) والثكل (2) يبين قيم التمهيد للتمهيد الاول للبيانات التي تم توليدها بعدد 80 وقيم السلسلة الحقيقية.

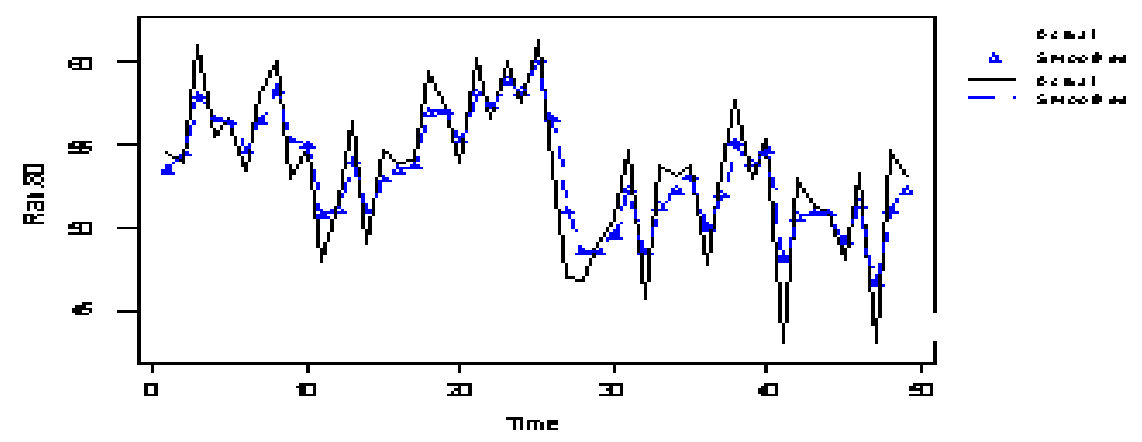

الشكل (2) يبين قيم التمهيد للتمهيد الاول بعد توليد (80) قيمة

وقيم السلسلة الحقيقية .

تم توليد بيانات بعدد 120 وذلك بنفس التباين مـع تغييرالمعدل للبيانات الحقيقية ومن ثم حذف اول اربعة وعشرين من البيانات واوسط اربعة وعشرين واخر اربعة وعشرين لغرض عدم التحيز ومن ثم تطبيق الجانب النظري عليها وكانت معادلة التمهيد لها $F_{t+1}^{\prime}=0.310 F_{t+1}+0.69 F_{t}^{\prime}$ حيـث كانـت قيمــة مربـع كـاي المحسـوبة ( الجدولية( 252=5.76 ) نجدها ايضاً اقل من الجدولية مما يدل على صحة التمهيد وكانت قيمة متوسط مربعات الخطأ ( MSD=17.0277 ) والثكل (3) يبين قيم 
التمهيد للتمهيد الاول (التمهيد المضـاعف) للبيانات التي تم توليدها بعدد 120 بعد تغيير قيمة المعدل وقيم السلسلة الحقيقية.

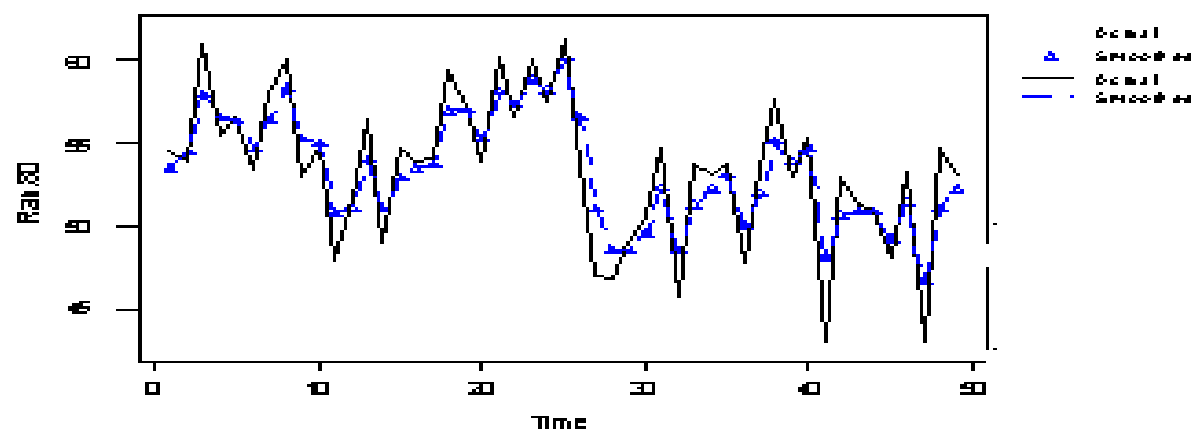

الثكل (3) يبين قيم التمهيد للتمهيد الاول للبيانات التي تم توليدها بعدد 120 وقيم

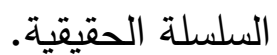

3- تم توليد بيانات بعدد 200 وذلك بنفس المعدل مـع تغيير التباين للبيانات الحقيقية ومن ثم حذف اول خمسين من البيانات واوسط خمسين واخر خمسين لغرض عدم التحيز ومن ثم تطبيق الجانب النظري عليها وكانت النتائج وكانت معادلة التمهيد لها علم الهيز

$F_{t+1}^{\prime}=0.41 F_{t+1}+0.59 F_{t}^{\prime}$

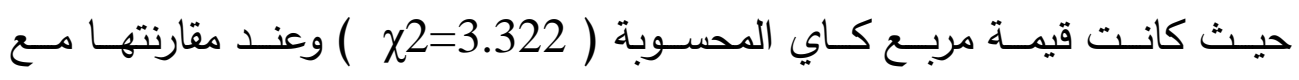
الجدولية(25.76=20 ) نجدها ايضاً اقل من الجدولية مما يدل على صحة التمهيد

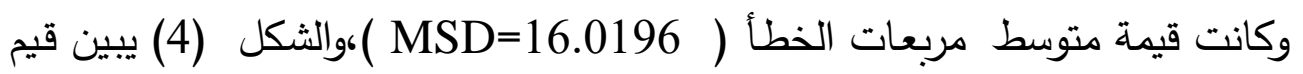
التمهيد للتمهيد الاول للبيانات التي تم توليدها بعدد 200 وقيم السلسلة الحقيقية. 


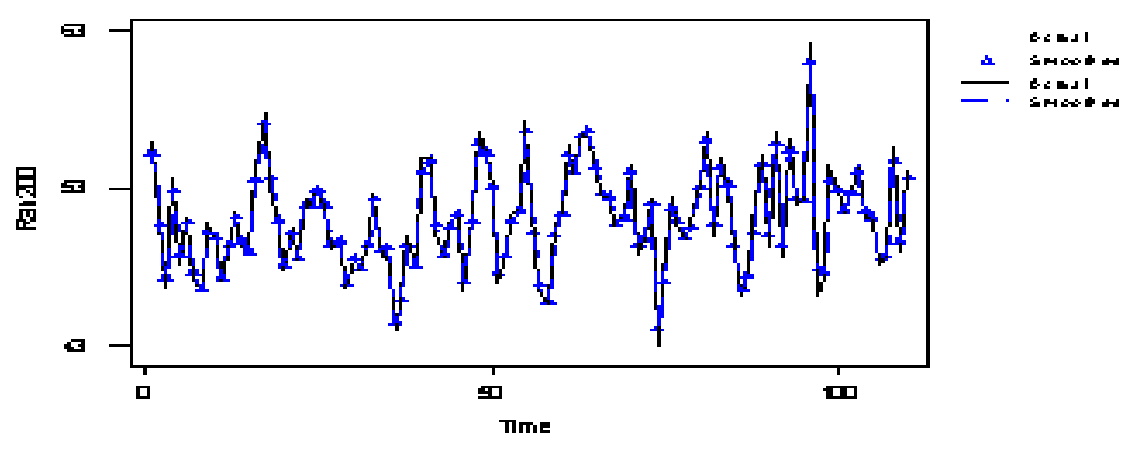

الشكل (4) يبين قيم التمهيد للتمهيد الاول للبيانات التي تم توليدها بعدد 200 وقيم السلسلة الحقيقية.

1. تم تقدير معلمات التمهيد للتمهيد الاول وذلك بالتشـابه مـع التمهيد الآسي المضـاعف كما هي في المعادلة (13) وتم اختبار صحة التمهيد بالنسبة إلى بيانات معدلات الرطوبة النسبية لمحافظة نينوى بالاعتماد على مربع كاي المحسوبة حيث كانت ( 3.4675 =2 ) وعند مقارنتها مـع قيمـة مربع كاي الجدولية (55.76= X )نجد إنها اقل من الجدولية عند مستوى معنوية (0.05) مما دل على صحة التمهيد ، وتمت المفاضلة بين التمهيد الاول والتمهيد للتمهيد الاول بالاعتماد على قيمة متوسط مربعات الخطاء حيـث كانـت قيمتهـا للتمهيد الاول (MSD=10.430 ) والتمهيد للتمهيد الاول (MSD=8.350) مما دل على ان التمهيد للتمهيد الاول افضل من

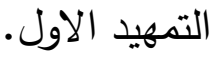
2. تم عمل محاكاة للبيانات (توليد بيانات ) وتطبيق الجانب النظري عليها وقياس صحة التمهيد بالاعتماد على مقياس مربع كاي ومتوسط مربعات الخطأ وكانت نتائج الاختبار تدل على صحة التمهيد للتمهيد الاول وبثلاث حالات من الأعداد العشوائية التي تم توليدها. 


$$
\text { 3. نوصــي باســخدام التمهيـد للتمهيـد الاول للبيانـات ، وذلـك لانــه يعـالج }
$$

\section{المصادرالأجنبية}

1- Douglas N; "Bayesian Confidence Intervals for Smoothing splines "JASA-DECEMBER 1988.v83 N404.

2- James D.Hamilton(1994); "Time Series Analysis 'princeton university press

3- Michael. I., Blake .A, 'A smoothing filter for Condensation' Proc $5^{\text {th }}$ European Conf.Computer Vision, Vol. 1 767-781 . 1998, misard@ robots.ox.ac.uk.

4- F.Celia,V.Balaji , Les M. , Amar .R, "Forecasting Women's Apparel Sales Using Mathematical Modeling"National Textile Center Annual Report ,November 2002.

5- $\quad$ Spyros .M, Steven C.W \&victor E,M(1983); ' Forecasting Methods and Application " second Edition, John wiley \&sons.

6- . Spyros .M, Steven C.W \&Rob J.Hyndman (1998); Forecasting Methods and Application " Third Edition, John wiley \&sons

$$
\text { المصادر العربية }
$$

1-راشد ، صفوان ناظم ، "تمهيد بيز لبعض النماذج الحركية الخطية " رسالة ماجستير

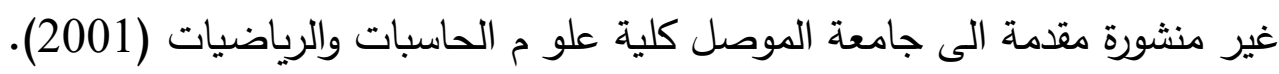
2-الجهاز المركزي للإحصاء " كراس التطور النوعي للإحصاء الزراعي لعام 1990. 